\title{
Nanometer-scale siRNA carriers incorporating peptidomimetic oligomers: physical characterization and biological activity
}

This article was published in the following Dove Press journal:

International Journal of Nanomedicine

10 May 2014

Number of times this article has been viewed

\author{
Yeliz Utku Konca' \\ Kent Kirshenbaum² \\ Ronald N Zuckermann ${ }^{3}$ \\ 'Department of Chemistry, \\ Koc University, Istanbul, Turkey; \\ 2Department of Chemistry, New York \\ University, New York, NY, ${ }^{3}$ Molecular \\ Foundry, Lawrence Berkeley National \\ Laboratory, Berkeley, CA, USA
}

\begin{abstract}
Synthetic short interfering RNA (siRNA) oligonucleotides can trigger the RNA interference pathway and lead to selective gene silencing. Despite considerable enthusiasm and investment, formidable challenges remain that may deter translating this breakthrough discovery into clinical applications. In particular, the development of efficient, nontoxic, nonimmunogenic methods for delivering siRNA in vivo has proven to be exceptionally challenging. Thorough analysis of the relationship between the structure and function of siRNA carrier systems, both in isolation and in complex with RNA, will facilitate the design of efficient nonviral siRNA delivery vehicles. In this study, we explore the relationship between the physicochemical characteristics and the biological activity of "lipitoid" compounds as potent siRNA delivery vehicles. Lipitoids are cationic peptidomimetic oligomers incorporating a peptoid and a phospholipid moiety. Lipitoids can associate with siRNA oligonucleotides and self-assemble into spherical lipitoid-based nanoparticles (LNPs), with dimensions that are dependent upon the medium and the stoichiometric ratio between the cationic monomers of the lipitoid and anionic siRNA oligonucleotides. The morphology, gene silencing efficiency, and cytotoxicity of the siRNA-loaded LNPs are similarly sensitive to the stoichiometry of the complexes. The medium in which the LNPs are formed affects the assembled cargo particles' characteristics such as particle size, transfection efficiency, and stability. Formation of the LNPs in the biological, serum-free medium OptiMEM resulted in LNPs an order of magnitude larger than LNPs formed in water, and were twice as efficient in siRNA transfection compared to LNPs formed in water. Inhibitor studies were conducted to elucidate the efficiency of lysosomal escape and the uptake mechanism of the siRNA-loaded LNPs. Our results suggest that these lipitoid-based, siRNA-loaded spherical LNPs are internalized through a lipid raft-dependent and dynaminmediated pathway, circumventing endosomal and lysosomal encapsulation. The lipitoid-siRNA nanospheres proved to be suitable platforms for investigating the critical parameters determining the efficiency of transfection agents, revealing the necessity for conducting characterization studies in biological media. The investigation of the LNP internalization pathway points to an alternative uptake route that bypasses the lysosome, explaining the surprisingly high efficiency of LNPs and suggesting that the uptake mechanism should be probed rather than assumed for the next generation of rationally designed transfection agents.
\end{abstract}

Keywords: lipitoid, siRNA delivery, therapeutic oligonucleotides, peptoid

\section{Introduction}

Short interfering ribonucleic acid (siRNA) can exert specific gene silencing effects in mammalian cells ${ }^{1,2}$ and thus have the potential to act as therapeutics for disease states that would otherwise be difficult to target via existing drugs. ${ }^{3}$ Early siRNA-based therapeutic regimens are currently in clinical trials, targeting more than a dozen diseases to 
date. ${ }^{4-6}$ Due to shortcomings regarding the biological stability and membrane permeability of naked siRNA, ${ }^{7}$ the routine clinical application of this promising therapeutic modality will rely upon the development of biocompatible molecular carrier systems that can efficiently deliver siRNAs to the target site. ${ }^{4,8,9}$ To date, a growing number of candidate molecules have been evaluated for their capability to facilitate intracellular nonviral uptake of siRNA.,10-17 An ongoing challenge is to attain improved understanding of the relationship between the chemical composition, the overall structure, and the biological function of these delivery molecules. ${ }^{18}$ Developing a robust structure-activity relationship for carrier molecules may enable rational design and lead to the discovery of guidelines for improving the potency, stability, specificity, and safety of siRNA delivery vehicles.

In this study, we investigate a molecular siRNA delivery system termed "lipitoid", which is composed of a cationic peptoid oligomer and a hydrophobic lipid moiety. ${ }^{19-21} \mathrm{~A}$ broad class of functionalized lipid materials shows promise as carriers for therapeutic siRNA. Not surprisingly, molecules similar to lipitoids, termed "lipidoids", have subsequently been investigated and shown to be effective not only in cell culture but also in higher organisms..$^{10,18,22-24}$ Ongoing studies in the rational design of these siRNA delivery vehicles have proposed designing smaller sized nanoparticle carriers that can penetrate the cells and support a mechanism of action entailing endocytic encapsulation and lysosomal escape, $6,18,25$ suggesting improved efficiency in delivery vehicles that can escape this pathway. ${ }^{25}$ Our molecule, containing a peptoid backbone, features many desirable properties of peptidomimetic oligomers, including facile solid-phase synthesis, ${ }^{26}$ access to exceptional side chain chemical diversity, ${ }^{27}$ and resistance to proteolytic degradation. ${ }^{28}$ Thus, peptoids constitute a modular oligomeric vehicle for siRNA delivery and provide an attractive platform to systematically investigate how alterations of particle formation parameters such as charge ratio, zeta potential, and morphology may influence transfection efficiency. Our earlier published studies have shown that this system is highly effective for oligonucleotide delivery, with efficiency comparable to, and in some cases superior to, commercial reagents such as Oligofectamine ${ }^{\mathrm{TM}}$, particularly for hard-to-transfect primary cell types. ${ }^{21,29}$ Here, our focus centers on investigating the origins of the functional potency. We show that this well-defined species, containing a nonamer hydrophilic peptoid backbone and two $\mathrm{C}_{12}$ lipid tails, associates with siRNA to form nanoparticle assemblies. In this study, we further explore the molecular and supramolecular structure-function relationships of this lipitoid-based nanoparticle (LNP) by probing the impact of complex formation conditions on the supramolecular morphology and the resulting effect on biological function. Our results reveal striking differences between both the physical properties and the biological function of LNPs formed in water and in biological media. The smallest LNPs were not necessarily the best performing ones, which may pose difficulties with intravenous injection but may ultimately improve the silencing efficiency of the LNPs by increasing the circulating half-life of these molecules in the bloodstream. ${ }^{8}$

The high silencing efficiency of the siRNA loaded LNPs may be due to the role of a different internalization pathway than commonly hypothesized for these molecules. Our results suggest a lipid raft/caveolae dependent internalization route, which bypasses the lysosome, and thus eliminates the need for a lysosomal escape step. Owing to their alternative internalization mechanism, these LNPs may further be exploited in the design of therapeutic biomaterials capable of intracellular targeting.

\section{Materials and methods Materials}

The lipitoid agent consists of a single high performance liquid chromatography-purified compound, DMPE(NaeNmpeNmpe), and was prepared as described earlier ${ }^{19}$ (dimirystoyl phosphatidyl-ethanolamine [DMPE]; $N$-(2aminoethyl)glycine [Nae]; $N$-[2-(4'-methoxyphenyl)ethyl] glycine [Nmpe]). The peptoid portion of the lipitoid molecule was synthesized on Rink amide resin, using an automated robotic synthesizer at room temperature. The lipid was subsequently manually conjugated to the oligomer at $60^{\circ} \mathrm{C}$. Following cleavage of the product from the solid support, the lipitoid was purified by high performance liquid chromatography and characterized by liquid chromatography-mass spectrometry. Green fluorescent protein (GFP)-22 siRNA (sense strand sequence GCAAGCUGACCCUGAAGUUCAU, molecular weight 17,001.9) and AllStars negative control siRNA (proprietary sequence, catalog number 1027280 , molecular weight 14,857.1) were obtained from Qiagen (Venlo, the Netherlands). NM2C5 human breast cancer cells and NM2C5-GFP cell lines were obtained from American Type Culture Collection (Manassas, VA, USA). U2OS human osteosarcoma cells and U2OS-GFP cell lines were a kind gift of the Pam Silver Lab (Harvard Medical School, Boston, MA, USA). Allophycocyanin (APC) conjugated annexin $\mathrm{V}$ was purchased from BD Biosciences (San Jose, CA, USA), propidium iodide (PI) was purchased from Roche (Basel, Switzerland), and all inhibitors were purchased from 
Sigma-Aldrich (St Louis, MO, USA). Cell culture reagents, including OptiMEM, Oligofectamine, Lipofectamine and RNAiMax were obtained from Life Technologies (Carlsbad, CA, USA). OptiMEM is an optimized modification of the serum-free Modified Eagle's Medium.

\section{Formation of lipitoid-siRNA complexes}

Samples of lipitoid and siRNA complexes were prepared by combining siRNA ( $20 \mu \mathrm{M}$ in Qiagen siRNA Suspension Buffer) and $500 \mu \mathrm{M}$ lipitoid solution prepared in distilled water. The solutions were warmed to room temperature prior to complex formation. As a sample calculation, for generation of LNPs at 3/1 (positive/negative) charge ratio, $5 \mu \mathrm{L} 20 \mu \mathrm{M}$ siRNA was diluted in $95 \mu \mathrm{L}$ OptiMEM, and in a separate tube, $7.5 \mu \mathrm{L} 500 \mu \mathrm{M}$ lipitoid was diluted in 92.5 $\mu \mathrm{L}$ OptiMEM. The two solutions were mixed by gentle aspiration, resulting in a complex formation solution composed of $500 \mathrm{nM}$ siRNA and $18.75 \mu \mathrm{M}$ lipitoid in a total volume of $200 \mu \mathrm{L}$ in OptiMEM. The amounts of siRNA were kept fixed, and the amount of lipitoid was adjusted as required for the other charge ratios used. Following incubation for 10 minutes at room temperature with occasional agitation, the resulting LNP complexes were used immediately for transfection, scanning electron microscopy (SEM) analysis, dynamic light scattering (DLS) imaging, zeta potential measurements, or atomic force microscopy (AFM) imaging.

\section{Transfection experiments}

U2OS and NM2C5 cells were counted and plated 24 hours prior to transfection at a concentration that would afford $70 \%-80 \%$ cell density at the time of the experiment. Cells were typically plated in 6-well plates in $2 \mathrm{~mL}$ growth medium. On the day of transfection, the cells were washed twice with $1 \mathrm{~mL}$ phosphate buffered saline (PBS) and once with $1 \mathrm{~mL}$ OptiMEM. The cells were then incubated in $1.8 \mathrm{~mL}$ OptiMEM at $37^{\circ} \mathrm{C}$ while the complexes were being prepared. As soon as the complexes of lipitoid and siRNA were formed as described, $200 \mu \mathrm{L}$ of the LNP-containing solution was gently added onto cells being incubated in $1.8 \mathrm{~mL}$ OptiMEM, resulting in final concentrations of $50 \mathrm{nM}$ siRNA and $1.875 \mu \mathrm{M}$ lipitoid (for LNPs formed at $3 / 1$ charge ratio). The cells were incubated in the presence of LNPs at $37^{\circ} \mathrm{C}$ under $5 \%$ carbon dioxide for 20 minutes before $500 \mu \mathrm{L}$ of $50 \%$ fetal bovine serum (FBS) was added to each well to achieve the normal growth conditions of $10 \%$ serum. The cells were then incubated for 48 hours prior to analysis by fluorescence assisted cell sorting
(FACS). Negative controls consisted of nontreated cells, cells treated only with lipitoid, and cells treated only with siRNA (at $50 \mathrm{nM}$ transfection concentration). Positive controls were performed with Oligofectamine, Lipofectamine ${ }^{\circledR}$, and RNAiMAX ${ }^{\mathrm{TM}}$. Transfections with the commercial transfection reagents were carried out as instructed in the manufacturers' protocols, with siRNA concentrations in the 20-200 nM range.

\section{Preparing SEM samples}

Silica wafers $(5 \times 7 \mathrm{~mm})$ were purchased from Ted Pella (Redding, CA, USA) and were plasma treated in a plasma cleaner for 3 minutes to remove organic contaminants. Solutions containing the formed complexes were transferred onto plasma-cleaned silica wafers. A volume of 10 $\mu \mathrm{L}$ complex solution was sufficient to evenly cover the surface of the wafer. The solution was incubated on the silica surface for 5 minutes at room temperature. Generally, the complexes were incubated for 5 minutes under ambient conditions in solution while complexes were being formed, then incubated for an additional 5 minutes on the wafer, which provided a total complex formation time consistent with transfection and DLS experiments (10 minutes). The excess solution was then removed by tapping the side of the wafer onto a clean laboratory cleaning tissue. The wafer was twice rinsed with double distilled water to remove remaining salts that could interfere with SEM analysis. The sample was then dried further at room temperature and atmospheric pressure to complete dryness. The sample was analyzed under a Gemini Ultra-55 analytical scanning electron microscope (Zeiss, Oberkochen, Germany) at 1 $\mathrm{kV}$ beam voltage using either an in-lens or SE2 detector (Zeiss).

\section{FACS analysis}

Growth medium was aspirated from the cell culture plates, and cells were washed once with $1 \mathrm{~mL}$ PBS before being trypsinized until they detached from the growing plate. The detached cells were collected in a $15 \mathrm{~mL}$ tube and pelleted by centrifugation at $1,000 \mathrm{rpm}$ for 5 minutes. The trypsin solution was removed, and cells were washed twice in 2 mL PBS. Finally, the cells were counted and resuspended in PBS at a concentration of $10^{6}$ cells $/ \mathrm{mL}$. The prepared samples were analyzed for fluorescence intensity by FACS. FACS data were collected on a BD FACS ARIA2 flow cytometer (BD Biosciences) equipped with $488 \mathrm{~nm}$ and 633 $\mathrm{nm}$ lasers and suitable band pass filters. GFP fluorescence was detected by utilizing the $488 \mathrm{~nm}$-laser with a $510 / 30$ 
bp filter, allowing monitoring of emission detection at 515-545 nm.

\section{Cellular viability tests}

PI staining was performed in order to determine cell viability and to exclude apoptosis-inducing mechanisms. In addition, annexin $\mathrm{V}$ was used to quantify apoptosis in tandem. APC-conjugated annexin V was applied to treated cells as described, ${ }^{30}$ and apoptosis and cell death was quantified by FACS through quantification of APC and PI, respectively. Lipitoid-siRNA complexes were formed at varying charge ratios in OptiMEM, using GFP-targeting siRNA (or nontargeting control siRNA). Silencing results for the targeted GFP expression were analyzed in GFP-expressing U2OS cells 48 hours posttransfection by FACS. All results are averages of at least three experiments, and silencing values are normalized with respect to nontreated cells as a control. Cytotoxicity was assessed by PI staining and analyzed by FACS.

\section{Inhibitor studies}

U2OS-GFP cells were transfected with a $3 / 1$ charge ratio of lipitoid:GFP siRNA complex, while being incubated with various internalization inhibitors. The complexes were formed for 10 minutes, and cells were preincubated with the inhibitors as was described in literature. ${ }^{31,32}$ Briefly, cells were incubated with $0.1 \%$ sodium azide $\left(\mathrm{NaN}_{3}\right)$ and $50 \mathrm{mM}$ 2-D-deoxyglucose (2DDG) for 1 hour, or with $50 \mu \mathrm{M}$ chloroquine for 30 minutes, or with $5 \mathrm{mM}$ methyl$\beta$-cyclodextrin (m $\beta \mathrm{cd}$ ) for 15 minutes, or with $10 \mu \mathrm{g} / \mathrm{mL}$ chlorpromazine for 15 minutes, or with $200 \mu \mathrm{M}$ genistein for 1 hour, or with $80 \mu \mathrm{M}$ dynasore for 1 hour. The cells were then coincubated with the complexes and inhibitors for 20 minutes. The medium containing the LNPs and inhibitors was removed, and following a PBS wash, fresh OptiMEM and FBS was added, yielding a final regular growth medium containing 10\% serum. GFP expression by cells was quantified by FACS in 48 hours. All experiments were performed three times with the exception of incubation on ice.

\section{AFM imaging}

Complexes were prepared as described above and deposited on a piece of freshly cleaved mica. The solution was incubated for 5 minutes on the mica surface for complete attachment. Excess solution was removed with a gentle stream of nitrogen gas and the sample was imaged immediately in AC mode by an Asylum MFP-3D atomic force microscope (Asylum
Research, Santa Barbara, CA, USA), equipped with a Multi$75 \mathrm{Al}$ tip.

\section{DLS imaging and zeta potential measurements}

Complexes were formed as described, placed into a Malvern Zetasizer nanoseries DTS 1061 capillary cell (Malvern Instruments, Malvern, UK), and analyzed with a Malvern Zetasizer Nano instrument at $25^{\circ} \mathrm{C}$. A Polybead Sampler Kit III (Polysciences, Warrington, PA, USA) was used for standardization in DLS measurements.

\section{Results and discussion Biological activity Lipitoid-siRNA stoichiometry}

The overall net charge of cationic carrier systems for oligonucleotides is one of the critical parameters determining the efficiency of transfection. ${ }^{33}$ In order to investigate the influence of charge on the lipitoid-siRNA complexes, samples were prepared at various charge ratios by varying the amount of lipitoid and keeping the amount of siRNA constant. The primary amine groups on the Nae sidechains and the secondary amine on the linker between the lipid and the peptoid portion of the lipitoid contribute to the net positive charge of the lipitoid, yielding three overall positive charges per lipitoid molecule (Figure 1).

Charge ratios of the formed LNPs were calculated by evaluating the ratio of the net positive charges on the lipitoid and the negative charges on the siRNA phosphodiester backbone (see the Materials and methods section for a sample calculation). LNPs were formed between the lipitoid and siRNA oligos targeting the GFP gene. Transfection experiments were carried out in two different human carcinoma cell lines, U2OS and NM2C5 expressing GFP, in order to rule out cell type specific response. Silencing efficiency was determined by quantifying the downregulation of GFP through FACS.

Lipitoid-siRNA particles formed between $0.5 / 1$ and 3/1 charge ratio in OptiMEM exhibited the most potent silencing efficiency ( $>90 \%$ of GFP) in U2OS cells (Figure 2). The ANOVA test was significant for silencing, with $P<0.001$. The significantly different values resulting from Bonferroni corrected $t$-tests are marked on the graph for $\%$ GFP expression results (Figure 2). Previously, we had confirmed that this level of silencing efficiency is significantly greater than that of Oligofectamine, ${ }^{21}$ with enhanced downregulation potency as evaluated by quantifying targeted mRNA levels and the targeted protein level of endogenous, physiologically 


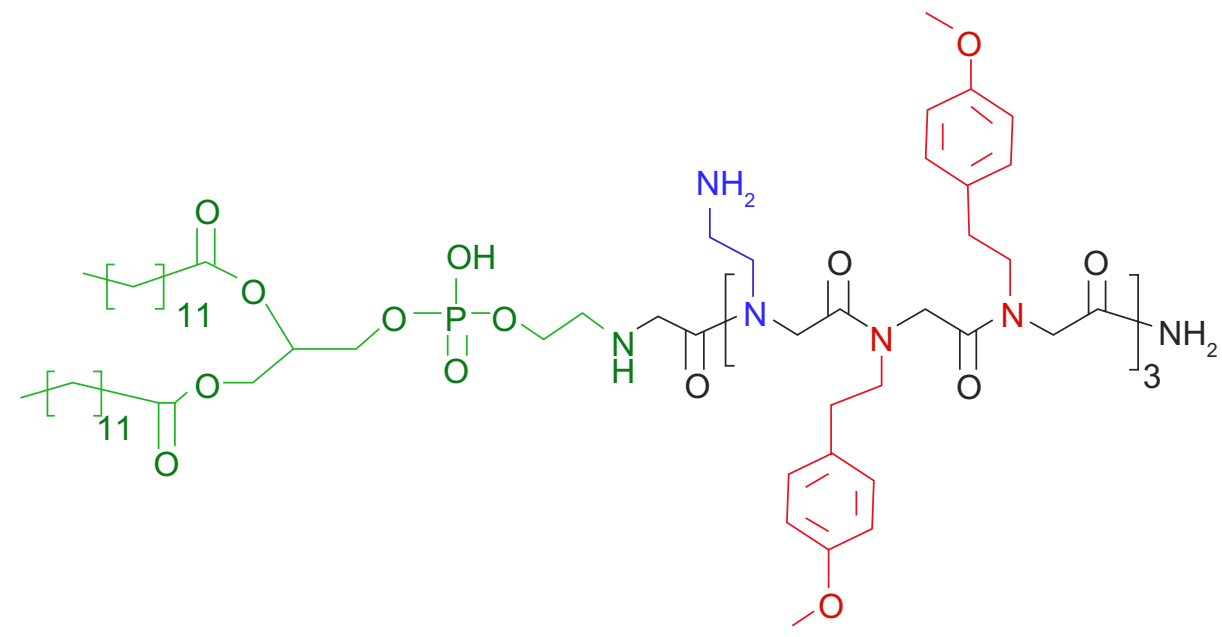

Figure I Chemical structure of the lipitoid.

Notes: The molecule is composed of a cationic, hydrophilic nonamer peptoid of the sequence (NaeNmpeNmpe) 3 and a double CI2 lipid tail based on dimirystoyl phosphatidyl-ethanolamine (DMPE; shown in green); $\mathrm{N}$-(2-aminoethyl)glycine (Nae; shown in blue); $\mathrm{N}$-[2-(4'-methoxyphenyl)ethyl]glycine (Nmpe; shown in red).

Abbreviation: DMPE, dimirystoyl phosphatidyl-ethanolamine.

relevant genes. Since previous cytotoxicity tests with 3-(4,5-dimethylthiazol-2-yl)-2,5-diphenyltetrazolium bromide (the MTT assay) showed no significant cytotoxicity, and there was no observable morphology change of the tested cells, ${ }^{21}$ toxicity was further probed by PI staining and later tested for early signs of apoptosis using annexin $\mathrm{V}$. The

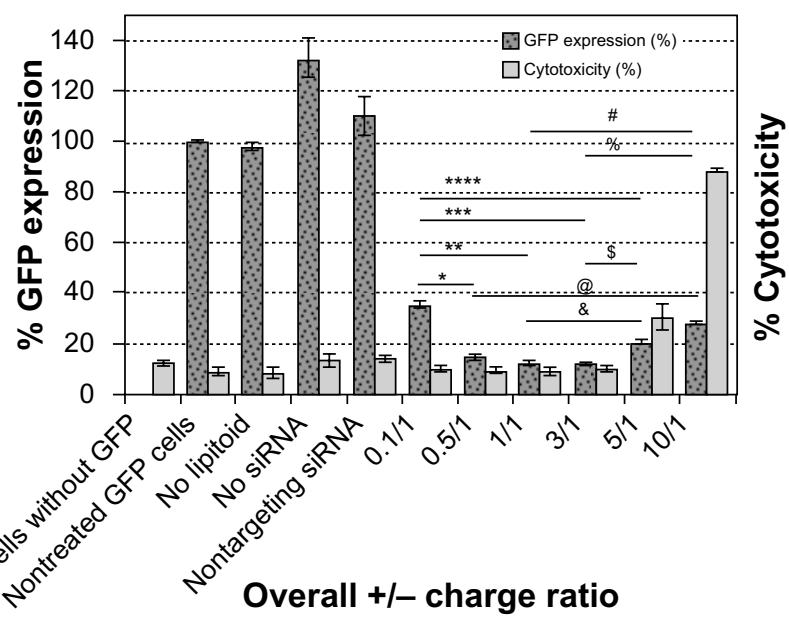

Figure $\mathbf{2}$ The effect of charge ratio on silencing efficiency and cytotoxicity. Notes: The most potent silencing and the lowest cytotoxicity were observed with complexes formed at charge ratios between $0.5 / I$ and $3 / I$, yielding silencing as high as $90 \%$ and cytotoxicity as low as $10 \%$ in U2OS cells. Charge ratios lower than $0.5 / I$ did not result in efficient transfection, whereas charge ratios higher than $3 / 1$ resulted in elevated cytotoxicity. All charge ratios had apoptosis rates similar to the untreated control cells (data not shown). The siRNA was used at $50 \mathrm{nM}$ concentration. ANOVA test was significant for both silencing activity and cytotoxicity. The significantly different values resulting from Bonferroni corrected $t$-tests are marked on the graph for \% GFP expression results. For cytotoxicity, the 5/I LNPs are significantly more toxic than all other LNPs except the 10/I LNPs, and the 10/I LNPs are significantly more toxic than all of the other tested LNPs. *, *w, ****, *w***, \&, @, \$, \%, \# indicate significantly different pairs, with $P<0.01$.

Abbreviations: GFP, green fluorescent protein; LNPs, lipitoid-based nanoparticles; siRNA, short interfering RNA. lowest cytotoxicity was observed for complexes formed at charge ratios between $0.5 / 1$ and $3 / 1$ (Figure 2). Cytotoxicity increased significantly at charge ratios above $3 / 1$, with a $5 / 1$ charge ratio evoking significant toxicity to treated cells and a 10/1 charge ratio leading to the death of most cells. For cytotoxicity, ANOVA tests and Bonferroni corrected $t$-tests indicated that the 5/1 LNPs were significantly more toxic than all other LNPs except the 10/1 LNPs, and the 10/1 LNPs were significantly more toxic than all of the other tested LNPs. Annexin V assay was performed to confirm that the cell death was not the outcome of induced apoptosis. The apoptosis rate for all the tested samples, including the nontreated control cells, remained at $1 \%-2 \%$, suggesting that none of the tested charge ratios contributed to a significant increase in apoptosis of the cells (data not shown). The 3/1 charge ratio represented the best performing charge ratio, and similar results were observed in NM2C5 cells (Figure S1), as toxicity was not evident for LNPs formed at or below a charge ratio of $3 / 1$. Positive controls conducted with Oligofectamine, Lipofectamine, and RNAiMAX indicated that the 3/1 charge ratio LNPs were comparable to, and in some cases better than, commercial transfection reagents (Figure S2). Further experiments were conducted by forming the lipitoid-siRNA LNPs at 3/1 charge ratio unless stated otherwise.

\section{Structural characteristics of the complexes}

\section{Lipitoid-siRNA nanoparticle morphology}

In order to ascertain the nanometer-scale morphology of the active siRNA/lipitoid assemblies of 3/1 charge ratio, these complexes were analyzed by DLS, AFM, and SEM. 

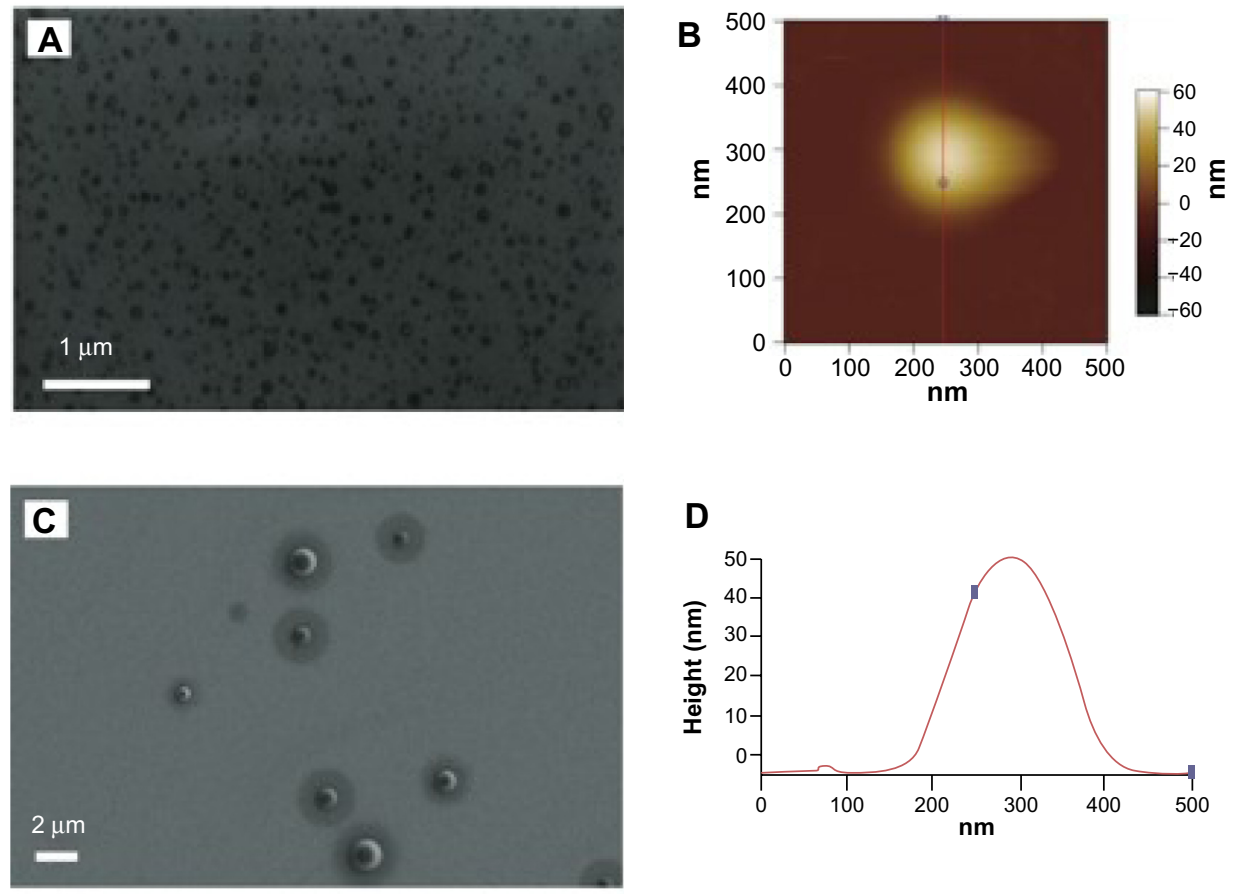

D



Figure 3 Lipitoid and siRNA self-assemble to form spherical LNPs in water.

Notes: LNPs grow in size when formed in the biological medium OptiMEM (Life Technologies, Carlsbad, CA, USA); (A) SEM image of lipitoid-siRNA nanoparticles in water showing circular LNPs; (B) AFM image of LNPs in water, confirming spherical shape; (C) Lipitoid-siRNA LNPs in OptiMEM are larger, submicrometer scale complexes; (D) Cross-section of the image in (B) showing the maximum height of the sphere at $50 \mathrm{~nm}$ and width at half-height at $150 \mathrm{~nm}$. All LNPs were formed at $3 / 4$ charge ratio with $50 \mathrm{~nm}$ siRNA.

Abbreviations: AFM, atomic force microscopy; LNPs, lipitoid-based nanoparticles; SEM, scanning electron microscopy; siRNA, short interfering RNA.

Lipitoid-siRNA assemblies were initially formed in water as described in Materials and methods. SEM imaging of these assemblies revealed disk-shaped nanoparticles, with radii in the range of 20-80 nm (Figure 3A). SEM analysis of such soft matter may show slightly larger sizes, presumably due to slight flattening of the nanospheres on the silica surface as a result of water removal. To confirm the nanoparticle shapes and sizes in aqueous medium, the assemblies were also analyzed by DLS in water. The average hydrodynamic radius as determined by DLS was observed to be $34 \pm 2 \mathrm{~nm}$. AFM imaging confirmed that the complexes were indeed spherical (Figure 3B and D). Together, these results confirm that the lipitoid interacts with siRNA in water to assemble into spherical LNPs of about $34 \mathrm{~nm}$ in radius in water.

Biological studies, however, are seldom conducted in pure water. In order to evaluate a more biologically relevant
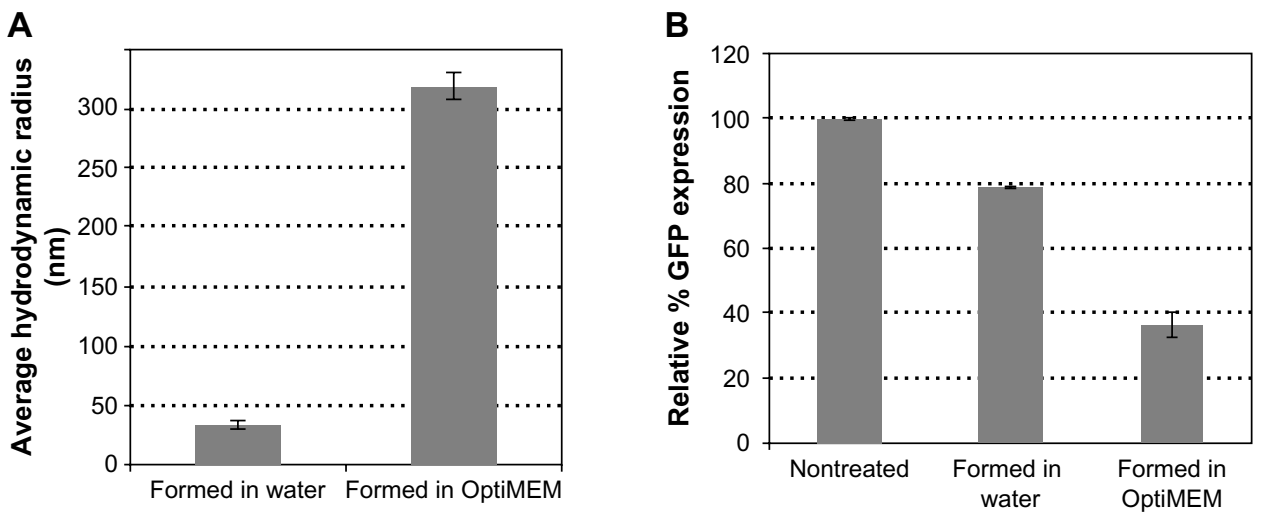

Figure 4 siRNA loaded LNP sizes depend on the medium in which they are formed.

Notes: Lipitoid-siRNA complexes were formed at 3/I charge ratio in water or in OptiMEM (Life Technologies, Carlsbad, CA, USA) with 50 nm siRNA.The sizes of the formed complexes were determined by DLS; (A) LNPs formed in water have a radii of $34 \pm 2 \mathrm{~nm}$, and the LNPs formed in the serum-free medium OptiMEM have radii averaging $320 \pm 1 \mathrm{I} \mathrm{nm}$; (B) The LNPs formed in water are less efficient than the LNPs formed in OptiMEM, only generating about $20 \%$ silencing effect as compared to the more than $60 \%$ silencing with the LNPs formed in OptiMEM.

Abbreviations: DLS, dynamic light scattering; GFP, green fluorescent protein; LNPs, lipitoid-based nanoparticles; siRNA, short interfering RNA. 

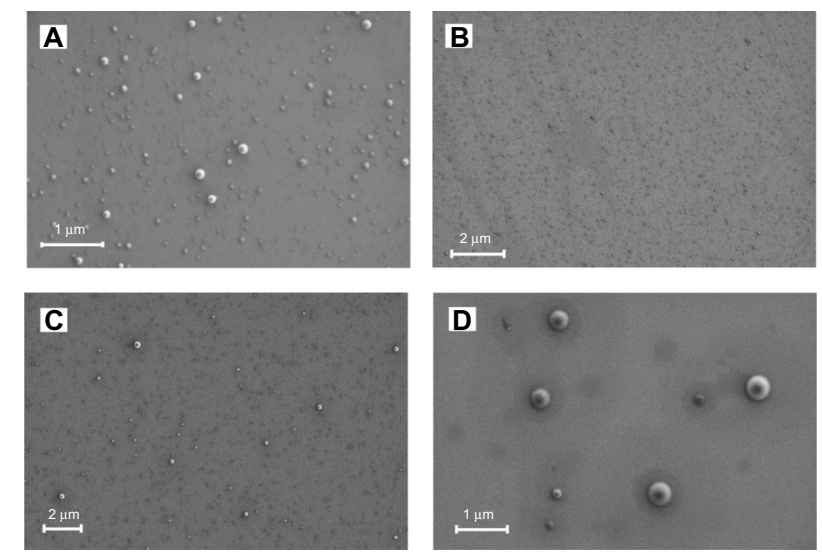

E

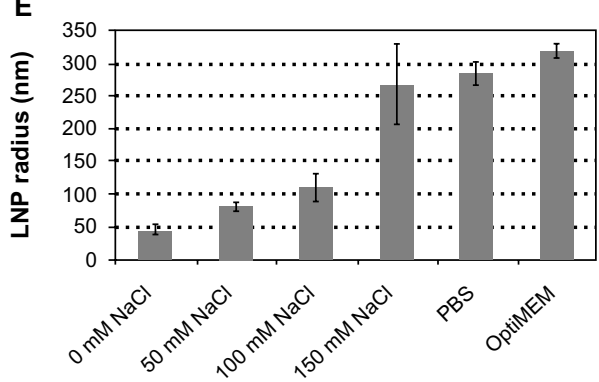

Figure 5 Lipitoid-siRNA nanoparticle sizes increase with increasing monovalent salt concentration.

Notes: (A) Lipitoid-siRNA nanoparticles formed in water; (B) Lipitoid-siRNA LNPs formed in $50 \mathrm{mM} \mathrm{NaCl}$; (C) Lipitoid-siRNA nanoparticles formed in $100 \mathrm{mM} \mathrm{NaCl}$; (D) Lipitoid-siRNA nanoparticles formed in $150 \mathrm{mM} \mathrm{NaCl}$; (E) Graph showing the sizes of LNPs in various media; All LNPs were formed at $3 /$ I $+/$ - charge ratio, using $50 \mathrm{~nm}$ siRNA in OptiMEM (Life Technologies, Carlsbad, CA, USA).

Abbreviations: LNPs, lipitoid-based nanoparticles; PBS, phosphate buffered saline; siRNA, short interfering RNA.

condition, lipitoid-siRNA complexes were formed in OptiMEM, a serum-free biological medium that is routinely used in cell culture experiments. SEM radii of the LNPs formed in OptiMEM were significantly larger, averaging $320 \pm 11 \mathrm{~nm}$ (Figures 3C and 4A). Similarly, spheres formed in the biological buffer PBS yielded complexes with an average hydrodynamic radius of $289 \pm 12 \mathrm{~nm}$ (Figure 5E). These order-of-magnitude enlargements of the LNP sizes revealed that the lipitoid-siRNA complexes respond very differently to biological media in comparison with water. The characterization of complexes formed in growth medium with supplemental serum was not carried out due to the complexity of the serum supplemented medium and the impossibility of probing the size of complexes in the presence of large serum proteins.

Owing to their order-of-magnitude smaller sizes, the LNPs formed in water were expected to be more efficient in gene silencing. Surprisingly, biological results showed that LNPs formed in water were not as efficient as the LNPs formed in OptiMEM, seen from the GFP expression of $79 \pm 0.2$ with the LNPs formed in water, as compared to
$36 \pm 6$ with the LNPs formed in OptiMEM. (Figure 4B). In order to study the effect of transferring the LNPs that were formed in water into a biological medium, the LNPs were formed in water as described in the Materials and methods and were then added to cell cultures growing in OptiMEM, following supplementation with $10 \%$ serum after transfection. The complexes grew in size when transferred into OptiMEM. However, these complexes were less stable and began to deteriorate within an hour of formation, as monitored by SEM, losing their spherical morphology (Figure S3). Gene silencing experiments conducted with these unstable complexes revealed that the silencing efficiency of the complexes formed in water was $66 \%$ lower than the silencing efficiency of the complexes formed in OptiMEM (data not shown). Thus, the LNPs formed in the biological medium OptiMEM were the largest in size and were also the best performing complexes as evaluated in cell culture. To further test the effect of serum on the biological activity of the LNPs, the best performing LNPs were used for transfection. LNPs formed at 3/1 charge ratio with a final concentration of $50 \mathrm{nM}$ siRNA were prepared in OptiMEM and then used to transfect siRNA, either into cells incubated in the serum-free OptiMEM or into cells incubated in serumsupplemented OptiMEM. Silencing activity was $50 \%$ with the transfection in serum-supplemented medium, as opposed to the $80 \%$ silencing in serum-free medium (Figure S4). This result reinforces the potency of LNPs, demonstrating that they can still generate a significant silencing effect even in the presence of serum.

\section{Complex size depends on ionic strength}

The origins of the LNP size enlargement in OptiMEM relative to water were investigated by probing the system in varying concentrations of salt. The concentrations of $\mathrm{NaCl}$ during complex formation were varied, at $0 \mathrm{mM}, 50 \mathrm{mM}, 100 \mathrm{mM}$, and $150 \mathrm{mM}$ (equivalent to the physiological monovalent salt concentration). Increasing the monovalent salt concentration resulted in the formation of LNPs with increasing spherical size. At $0 \mathrm{mM} \mathrm{NaCl}$ concentration the sizes of the nanospheres were homogeneous and averaged $r=47 \pm 6 \mathrm{~nm}$; at 50 $\mathrm{mM} \mathrm{NaCl}$ the LNPs grew to $r=85 \pm 3 \mathrm{~nm}$; at $100 \mathrm{mM} \mathrm{NaCl}$ the average was $r=110 \pm 20 \mathrm{~nm}$; at $150 \mathrm{mM} \mathrm{NaCl}$ most of the spheres were between $150 \mathrm{~nm}$ and $300 \mathrm{~nm}$ in diameter, with an average of $r=269 \pm 62 \mathrm{~nm}$ (Figure 5). As expected from previous results concerning the effect of ionic strength, ${ }^{34}$ within the tested concentration range of $0-150 \mathrm{mM}$, ionic strength is the main determinant of the complex sphere size - increasing salt concentration results in larger spheres. 
The major factor contributing to the size increase of the LNPs when formed in OptiMEM as opposed to in water is thus identified to be the monovalent salt concentration of the solution in which the LNPs are formed. The LNP sizes in $150 \mathrm{mM}$ $\mathrm{NaCl}(r=269 \pm 62 \mathrm{~nm})$ are almost identical to the LNP sizes in PBS $(r=289 \pm 12 \mathrm{~nm})$ and slightly smaller than the LNP sizes in OptiMEM ( $r=320 \pm 11 \mathrm{~nm}$ ) (Figure 5E). The slightly larger LNP size in OptiMEM may be attributed to protein binding or to the other numerous variables in OptiMEM (such as divalent cations, amino acids, etc). The decreased biological activity in the presence of serum may be due to formation of large aggregates, which were impossible to characterize by DLS, SEM, or AFM. This aggregation of the LNPs may be due to protein binding, whereby the resulting conglomerate is too large to stick onto and stay on mica or silica surfaces, larger than the limits of detection of DLS, and too large to be taken up into the cells in vitro.

\section{The effect of charge ratio on complex size and morphology}

To investigate the effect of charge ratio on the structure of lipitoid-siRNA LNPs, complexes of lipitoid and siRNA were formed at varying charge ratios of cationic lipitoid and anionic siRNA as described above. The sizes and morphologies of the LNPs varied significantly (Figure 6A-G). Sizes of the spherical LNPs increased with increasing charge ratio (Figure 6G and Table S1); however, at the elevated charge ratio of $10 / 1$, the spherical nature of the complexes was observed to be distorted (Figure 6F), and DLS readings were outside of the detectable range. These high charge ratio LNPs were also shown to be extremely toxic and less efficient in silencing (Figure 2). Despite the differences in size and morphology, the zeta potential of all complexes formed in OptiMEM was similar, in the range of -12 to -6 $\mathrm{mV}$ (Table S1). This phenomenon of slightly negative zeta potential in biological medium was also recently observed for other cationic cell penetrating systems. ${ }^{35}$ For comparison, the zeta potential of complexes formed in water was $+18.5 \mathrm{mV}$. Both in water and in OptiMEM, the zeta potential of the LNPs was within the -20 to $+20 \mathrm{mV}$ range, which is considered mostly neutral, and the differences within this range are not considered significant. OptiMEM is abundant in physiological salts. Since zeta potential is a relative measure of the charges of the particles within the solution with respect to the surrounding environment, the slightly negative zeta potential in OptiMEM may suggest that OptiMEM is a more negatively charged medium than the LNPs. In support of this analysis, the zeta potential of OptiMEM alone was
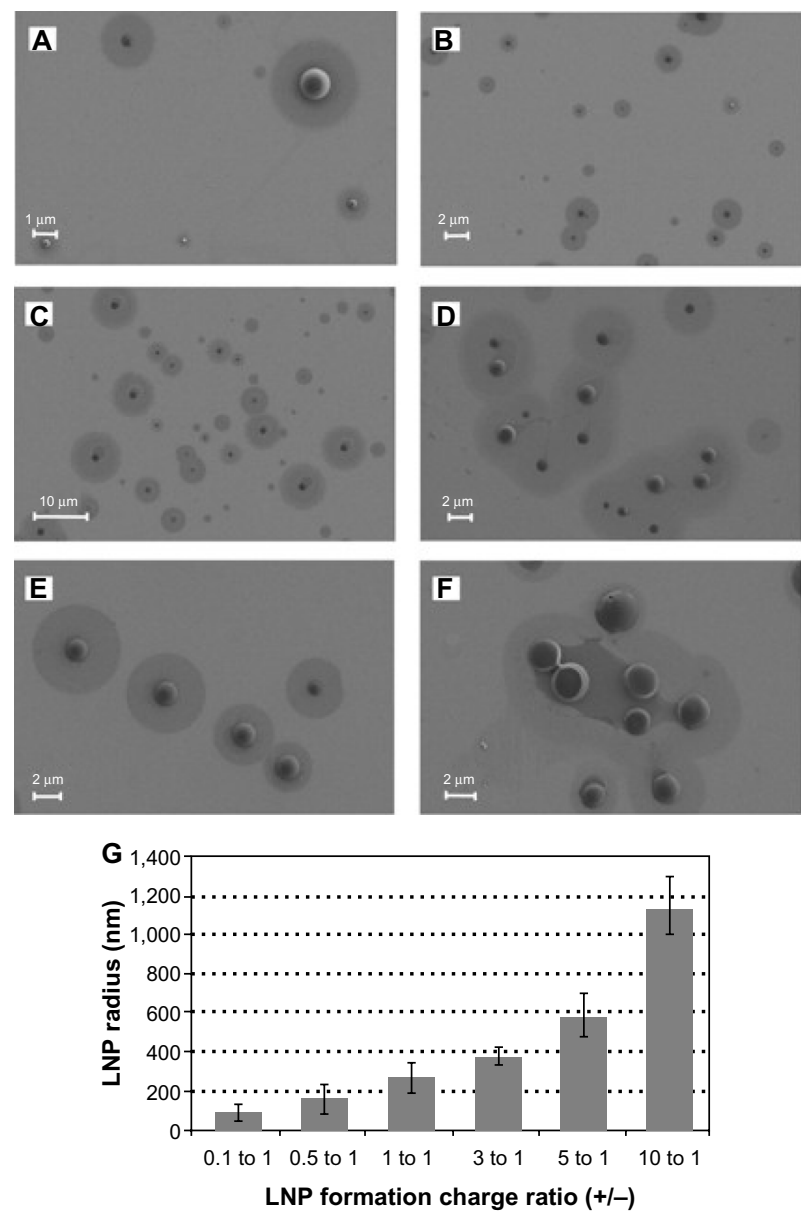

Figure 6 Lipitoid-siRNA complexes at varying charge ratios.

Notes: Images show lipitoid-siRNA LNPs formed at charge ratios of (A) $0.1 / 1$, (B) $0.5 / I$, (C) I/I, (D) 3/I, (E) 5/I, and (F) I0/I and analyzed by SEM; Average LNP radii are reported in the graph $(\mathbf{G})$; Nanoparticles formed at $0.1 /$ / charge ratio are the smallest but vary largely in size and shape while particles formed at 10/I charge ratio have grown a lot and started deteriorating and losing their spherical morphology. All LNPs were formed in OptiMEM with $50 \mathrm{~nm}$ siRNA.

Abbreviations: LNPs, lipitoid-based nanoparticles; SEM, scanning electron microscopy; siRNA, short interfering RNA.

measured to be $-30 \mathrm{mV}$. These observations illuminate the structure-function relationship between the complexes and their silencing effect. The charge ratio between the lipitoid and the siRNA is a crucial parameter in the formation of lipitoid-siRNA LNPs, affecting not only the size but also the morphology of the formed LNPs. Surprisingly, the charge ratio of LNPs does not seem to dramatically alter the zeta potential of the particles. The $3 / 1$ charge ratio LNPs provided optimal conditions for assembly between the lipitoid molecules and the siRNA oligos, producing spheres of a homogeneous size in the submicrometer scale, with LNP radii of about $34 \mathrm{~nm}$ in water and about $320 \mathrm{~nm}$ in biological media, with a mostly neutral zeta potential within the $\pm 20 \mathrm{mV}$ range, leading to outstanding efficiency in siRNA delivery and targeted gene downregulation, while exhibiting minimal toxicity. 


\section{Cellular uptake mechanisms}

Inhibiting the internalization pathway

of the lipitoid-siRNA complexes

Rational design of effective siRNA carrier systems can be possible only through knowledge of their cellular entry routes. The cellular uptake of delivery vehicles is infrequently studied, and it is often assumed that siRNA vehicles will be endocytosed and transported to the lysosome, following the "proton-sponge hypothesis". $8,36,37$ Recent data have revealed that this is not always the case and that some very widely studied transfection agents follow an uptake mechanism which bypasses endosomes and the lysosome, ${ }^{38-40}$ differing from the assumed internalization route. To reveal whether the cellular uptake mechanism of the lipitoid-siRNA complexes depends on lysosomal encapsulation and escape, we probed the internalization pathway of the best performing complexes in the presence of a set of chosen inhibitors acting at certain stages of various possible cell internalization pathways. GFPexpressing U2OS cell lines were targeted with GFP siRNA, and the inhibitors were used as described in the literature (see Materials and methods). The outcome of all inhibition tests was measured by quantification of the expressed GFP level by flow cytometry (Figure 7).

The most potent inhibitor was $\mathrm{m} \beta \mathrm{cd}$, which is known to deplete cholesterol in lipid rafts. ${ }^{41} \mathrm{~m} \beta \mathrm{cd}$ inhibition resulted in complete elimination of GFP silencing, suggesting a lipid raft-mediated uptake. The increase in GFP expression was dramatic and higher than the expected maximum level of $100 \%$ upon inhibiting the transfection with $\mathrm{m} \beta \mathrm{cd}$, which is a commonly observed trend with $\mathrm{m} \beta \mathrm{cd}$ blocking. ${ }^{16}$ To confirm that internalization is lipid raft mediated, we utilized dynasore, a reversible, noncompetitive inhibitor of dynamin 1 and dynamin2 GTPase. The use of dynasore also resulted

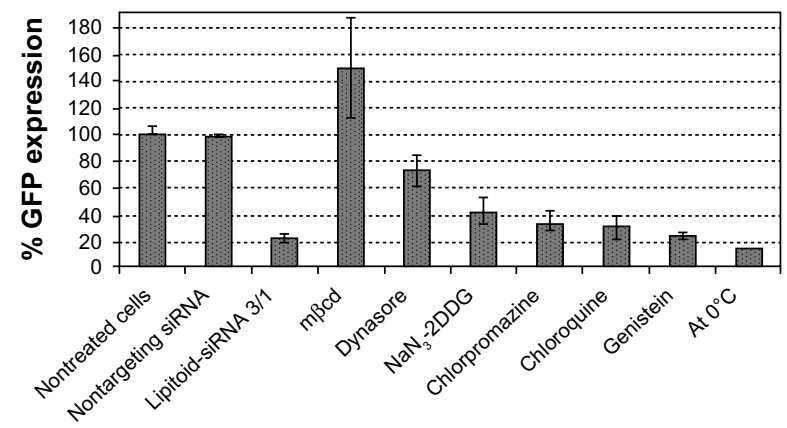

Figure 7 Inhibitors of internalization and their effects on lipitoid-siRNA complex uptake and subsequent silencing of GFP expression.

All LNPs were formed in OptiMEM with $50 \mathrm{~nm}$ siRNA and co-incubated with inhibitors in U2OS cells. Inhibitors were used as described in the literature.

Abbreviations: 2DDG, 2-D-deoxyglucose; GFP, green fluorescent protein; $\mathrm{m} \beta \mathrm{cd}$, methyl- $\beta$-cyclodextrin; siRNA, short interfering RNA. in dramatic inhibition of the LNP transfection activity as evident from the mere $25 \%$ silencing of GFP expression (Figure 7). Considering that GTPase activity of dynamin is essential for the budding of the caveolae from the plasma membrane, this result suggests that lipitoid-siRNA complexes are internalized through a dynamin- and lipid raft/caveolaedependent pathway. $\mathrm{NaN}_{3}$ and 2DDG were used in tandem to block ATP synthesis in the cell to test for the involvement of energy-dependent endocytosis. Uptake inhibition in the presence of $0.1 \% \mathrm{NaN}_{3}$ and $50 \mathrm{mM} 2 \mathrm{DDG}$ had little effect on the internalization of the complexes, suggesting that internalization is not energy-dependent. Chlorpromazine, a cationic amphiphatic drug, was used to block clathrinmediated endocytosis. Chlorpromazine, which incorporates into the lipid layer of the plasma membrane and increases lipid fluidity, hampering formation of invaginations, ${ }^{33}$ is a cationic amphiphatic drug typically used to block clathrinmediated endocytosis. Clathrin-mediated endocytosis was ruled out as the primary route of entry by the inefficiency of chlorpromazine as an inhibitor. Chloroquine is a widely used lysosomal inhibitor that acts by inhibiting the acidification of organelles. The use of chloroquine did not have a dramatic effect on LNP internalization, suggesting that neither lysosomal inhibition nor endosome escape plays a significant role on the internalization efficiency of lipitoid-siRNA LNPs. This result is in agreement with previous studies conducted with lipitoid-DNA uptake studies. ${ }^{32}$ The use of genistein, a nonspecific tyrosine kinase inhibitor, which mainly inhibits clathrin-mediated endocytosis, further confirms a nonclathrin-mediated cellular uptake of lipitoid-siRNA nanoplexes. The widely assumed "proton-sponge" hypothesis for the internalization of cationic siRNA delivery lipids is thus unlikely to be a primary mechanism for the internalization of lipitoid-siRNA complexes. The proton-sponge hypothesis is further ruled out by inhibition experiments performed by incubating the cells at $0^{\circ} \mathrm{C}$. The low temperature did not significantly block the internalization of these complexes, revealing that even when cellular processes are generally slowed and endocytosis is significantly reduced, the lipitoidsiRNA complex internalization is not halted.

In combination, these inhibition results suggest that there may be more than one possible route of internalization of these complexes. The major route may be a nonclathrin, nonendocytosis, lipid raft/caveolae-dependent, and dynaminmediated process. Alternatively, the lipitoid-siRNA based LNPs may also utilize an uptake route that bypasses both the lysosome and the endosome, which would consequently not be dependent on endosomal escape mechanisms. 
The results of this study reveal that lipitoid-siRNA nanospheres do not follow the classical endosome internalization pathway, which requires a lysosomal escape route. Instead, siRNA-loaded lipitoid-based LNPs are internalized through a clathrin-independent, dynamin and lipid raft-dependent pathway of internalization, which bypasses the lysosome. This route of internalization has recently been proposed as a distinct pathway of entry into the cell and requires the involvement of caveolae, dynamin, and actin. ${ }^{42}$ In agreement with our results, a recent finding with latex beads reveals that as the particle size increases from $50 \mathrm{~nm}$ to around $500 \mathrm{~nm}$, the route of internalization shifts from clathrin-mediated to caveolae-mediated uptake, bypassing the lysosome. ${ }^{43}$

Caveolae are invaginations on the plasma membranes of the cells, enriched in cholesterol and sphingolipids, and are not typically involved in classical endocytosis. ${ }^{44,45}$ Since they are involved in cell signaling pathways, however, caveolae are also rich in some cell-signaling receptors such as the folate receptor. ${ }^{45}$ Although caveolae are present in most normal tissues, they are overexpressed in some solid tumors. ${ }^{46}$ Since the discovery of tissue-specific, overexpressed caveolar molecules, it has been proposed that caveolae may be employed as a useful site for targeted gene delivery in vivo. ${ }^{46}$ The lipitoid-siRNA nanospheres may ultimately be suitable candidates for targeting caveolae-rich cancer cells, owing to their efficiency and route of cellular uptake.

\section{Conclusion}

The rational design of siRNA delivery systems can only be possible if the factors that influence the efficiency of these systems are properly described. In order to gain more knowledge about the structural characteristics of an efficient siRNA delivery system, we investigated the cellular uptake and gene silencing activity of lipitoid DMPE-(NaeNmpeNmpe) $)_{3}$ in complex with siRNA oligos.

Here we show that the lipitoid forms spherical complexes with siRNA, termed LNPs, in which the complex size strongly depends on the ionic strength of the medium. This parameter affects not only the size but also the morphology of the complexes, thereby leading to alterations in transfection efficiency. When LNPs formed in sterile distilled water are transferred into the biological growth medium OptiMEM, the spheres become enlarged by an order of magnitude, resulting in low transfection efficiency. When the lipitoid-siRNA LNPs are formed directly in the biological medium OptiMEM, the spheres are still an order of magnitude larger than the LNPs formed in distilled water; however, they result in higher silencing efficiency and virtually no cytotoxicity.
Further investigation of the lipitoid-siRNA LNP system in OptiMEM revealed that the stoichiometry of lipitoid to RNA (expressed as positive to negative charge ratio) was another critical parameter, affecting not only the size and morphology of the formed complexes, but also their transfection efficiency and cytotoxicity. Lipitoid-siRNA based LNPs formed at the charge ratio 3/1 in OptiMEM were determined to be the best performing complexes for this system. These LNPs, with average radii of $320 \pm 11 \mathrm{~nm}$ in OptiMEM, were not the smallest sized LNPs that could be obtained through the selfassembly of lipitoid and siRNA, but they resulted in up to $>90 \%$ silencing efficiency, with virtually no cytotoxicity. As a result, the lipitoid-siRNA LNPs were shown to be a defined system that could be fine-tuned and designed for optimized bioactivity. In vivo administration will require additional optimization, including a balance of attaining particle sizes that are sufficiently large to permit effective silencing benefiting from an increased blood circulation time, but sufficiently small to avert systemic difficulties such as diminished transport through the leaky tumor vasculature. ${ }^{47}$

The route of internalization of these LNPs into the cell was shown to bypass the lysosome, possibly through a lipid $\mathrm{raft} /$ caveolae-mediated and dynamin-dependent route, which does not utilize clathrin or endocytosis. This result suggests a possible explanation for the high efficiency of the LNPs, and indicates that an endosomal escape mechanism may not be manifest for every system. In fact, uptake mechanisms may be distinct for different systems, and the route of internalization should be probed instead of assumed. The observed route of internalization for the lipitoid-siRNA LNPs has recently been proposed as a distinct pathway of entry into the cell and requires the participation of caveolae, dynamin, and actin. ${ }^{42}$ This result may be beneficial in designing strategies that are based on targeting the caveolae, which are known to be overexpressed in certain tumorigenic tissues.

Currently, investigations of carrier systems are frequently performed in water; however, our study reveals that both the physical and the biological behavior of the siRNA cargo complexes are significantly different in biological medium in comparison to water. It is evident from our results that selecting for the smallest cargo vehicles may be counterproductive, as efficiency of transfection may increase with increasing LNP size. The route of cellular internalization may be different for each system, as shown for the LNPs presented here, and it should not be assumed that every siRNA delivery vehicle will be endocytosed and then transferred to the lysosome, from which the siRNA will escape. The results presented in this study will facilitate the rational design of 
siRNA delivery systems, exhibiting further enhancements in efficiency and cell-type specificity.

\section{Acknowledgments}

Portions of this work were performed as a User Project at the Molecular Foundry, Lawrence Berkeley National Laboratory, which is supported by the Office of Science, Office of Basic Energy Sciences of the Department of Energy under Contract No DE-AC02-05CH11231. The support of the National Science Foundation (NSF), through award CHE-\#1152317 to KK, is gratefully acknowledged. This work would not be possible without the Horizon Travel Award for Research, the Margaret and Herman Sokol Travel/Research Award, and MacCracken Full Fellowship awarded by New York University to YUK.

\section{Disclosure}

The authors report no conflicts of interest in this work.

\section{References}

1. Elbashir SM, Harborth J, Lendeckel W, Yalcin A, Weber K, Tuschl T. Duplexes of 21-nucleotide RNAs mediate RNA interference in cultured mammalian cells. Nature. 2001;411(6836):494-498.

2. Elbashir SM, Lendeckel W, Tuschl T. RNA interference is mediated by 21- and 22-nucleotide RNAs. Genes Dev. 2001;15(2):188-200.

3. Haussecker D. The business of RNAi therapeutics. Hum Gene Ther. 2008;19(5):451-462.

4. Burnett JC, Rossi JJ. RNA-based therapeutics: current progress and future prospects. Chem Biol. 2012;19(1):60-71.

5. Burnett JC, Rossi JJ, Tiemann K. Current progress of siRNA/shRNA therapeutics in clinical trials. Biotechnol J. 2011;6(9):1130-1146.

6. Resnier P, Montier T, Mathieu V, Benoit JP, Passirani C. A review of the current status of siRNA nanomedicines in the treatment of cancer. Biomaterials. 2013;34(27):6429-6443.

7. Yoo JW, Irvine DJ, Discher DE, Mitragotri S. Bio-inspired, bioengineered and biomimetic drug delivery carriers. Nat Rev Drug Discov. 2011;10(7):521-535.

8. Whitehead KA, Langer R, Anderson DG. Knocking down barriers: advances in siRNA delivery. Nat Rev Drug Discov. 2009;8(2):129-138.

9. Shi J, Votruba AR, Farokhzad OC, Langer R. Nanotechnology in drug delivery and tissue engineering: from discovery to applications. Nano Lett. 2010;10(9):3223-3230.

10. Akinc A, Goldberg M, Qin J, et al. Development of lipidoid-siRNA formulations for systemic delivery to the liver. Mol Ther. 2009;17(5): 872-879.

11. Patil ML, Zhang M, Taratula O, Garbuzenko OB, He H, Minko T. Internally cationic polyamidoamine PAMAM-OH dendrimers for siRNA delivery: effect of the degree of quaternization and cancer targeting. Biomacromolecules. 2009;10(2):258-266.

12. Aigner A. Cellular delivery in vivo of siRNA-based therapeutics. Curr Pharm Des. 2008;14(34):3603-3619.

13. Andersen MØ, Howard KA, Paludan SR, Besenbacher F, Kjems J. Delivery of siRNA from lyophilized polymeric surfaces. Biomaterials. 2008;29(4):506-512.

14. Beh CW, Seow WY, Wang Y, et al. Efficient delivery of Bcl-2-targeted siRNA using cationic polymer nanoparticles: downregulating mRNA expression level and sensitizing cancer cells to anticancer drug. Biomacromolecules. 2009;10(1):41-48.

15. Breunig M, Hozsa C, Lungwitz U, et al. Mechanistic investigation of poly(ethylene imine)-based siRNA delivery: disulfide bonds boost intracellular release of the cargo. $J$ Control Release. 2008;130(1):57-63.
16. Crombez L, Aldrian-Herrada G, Konate K, et al. A new potent secondary amphipathic cell-penetrating peptide for siRNA delivery into mammalian cells. Mol Ther. 2009;17(1):95-103.

17. Green JJ, Langer R, Anderson DG. A combinatorial polymer library approach yields insight into nonviral gene delivery. Acc Chem Res. 2008;41(6):749-759.

18. Semple SC, Akinc A, Chen J, et al. Rational design of cationic lipids for siRNA delivery. Nat Biotechnol. 2010;28(2):172-176.

19. Huang CY, Uno T, Murphy JE, et al. Lipitoids - novel cationic lipids for cellular delivery of plasmid DNA in vitro. Chem Biol. 1998;5(6): 345-354.

20. Lobo BA, Vetro JA, Suich DM, Zuckermann RN, Middaugh CR. Structure/function analysis of peptoid/lipitoid:DNA complexes. J Pharm Sci. 2003;92(9):1905-1918.

21. Utku Y, Dehan E, Ouerfelli O, et al. A peptidomimetic siRNA transfection reagent for highly effective gene silencing. Mol Biosyst. 2006;2(6-7):312-317.

22. Akinc A, Zumbuehl A, Goldberg M, et al. A combinatorial library of lipid-like materials for delivery of RNAi therapeutics. Nat Biotechnol. 2008;26(5):561-569.

23. Love KT, Mahon KP, Levins CG, et al. Lipid-like materials for lowdose, in vivo gene silencing. Proc Natl Acad Sci U S A. 2010;107(5): 1864-1869.

24. Chen D, Love KT, Chen Y, et al. Rapid discovery of potent siRNAcontaining lipid nanoparticles enabled by controlled microfluidic formulation. J Am Chem Soc. 2012;134(16):6948-6951.

25. Sahay G, Querbes W, Alabi C, et al. Efficiency of siRNA delivery by lipid nanoparticles is limited by endocytic recycling. Nat Biotechnol. 2013;31(7):653-658.

26. Zuckermann RN, Kerr JM, Kent SBH, Moos WH. Efficient method for the preparation of peptoids [oligo(N-substituted glycines)] by submonomer solid-phase synthesis. J Am Chem Soc. 1992;114(26):10646-10647.

27. Sun J, Zuckermann RN. Peptoid Polymers: A Higher Designable Bioinspired material. ACS Nano. 2013:7;4715-4732.

28. Miller SM, Simon RJ, Ng S, Zuckermann RN, Kerr JM, Moos WH. Comparison of the proteolytic susceptibilities of homologous L-amino acid, D-amino acid, and N-substituted glycine peptide and peptoid oligomers. Drug Dev Res. 1995;35(1):20-32.

29. Schiffmacher AT, Keefer CL. Optimization of a lipitoid-based plasmid DNA transfection protocol for bovine trophectoderm CT-1 cells. In Vitro Cell Dev Biol Anim. 2012;48(7):403-406.

30. Zamai L, Canonico B, Luchetti F, et al. Supravital exposure to propidium iodide identifies apoptosis on adherent cells. Cytometry. 2001;44(1):57-64.

31. Gratton SE, Ropp PA, Pohlhaus PD, et al. The effect of particle design on cellular internalization pathways. Proc Natl Acad Sci U SA. 2008;105(33):11613-11618.

32. Murphy JE, Uno T, Hamer JD, Cohen FE, Dwarki V, Zuckermann RN. A combinatorial approach to the discovery of efficient cationic peptoid reagents for gene delivery. Proc Natl Acad Sci U SA. 1998;95(4):1517-1522.

33. Masotti A, Mossa G, Cametti C, et al. Comparison of different commercially available cationic liposome-DNA lipoplexes: Parameters influencing toxicity and transfection efficiency. Colloids Surf B Biointerfaces. 2009;68(2):136-144.

34. Schneider G, Decher G. Functional core/shell nanoparticles via layerby-layer assembly. investigation of the experimental parameters for controlling particle aggregation and for enhancing dispersion stability. Langmuir. 2008;24(5):1778-1789.

35. van Asbeck AH, Beyerle A, McNeill H, et al. Molecular parameters of siRNA-cell penetrating peptide nanocomplexes for efficient cellular delivery. ACS Nano. 2013;7(5):3797-3807.

36. Behr JP. The proton sponge: a trick to enter cells the viruses did not exploit. Chimia Int J Chem. 1997;51:34-36.

37. Boussif O, Lezoualc'h F, Zanta MA, et al. A versatile vector for gene and oligonucleotide transfer into cells in culture and in vivo: polyethylenimine. Proc Natl Acad Sci U S A. 1995;92(16): 7297-7301. 
38. Gabrielson NP, Pack DW. Efficient polyethylenimine-mediated gene delivery proceeds via a caveolar pathway in HeLa cells. J Control Release. 2009;136(1):54-61.

39. Chung YC, Cheng TY, Young TH. The role of adenosine receptor and caveolae-mediated endocytosis in oligonucleotide-mediated gene transfer. Biomaterials. 2011 Jul;32(19):4471-4480.

40. Perez AP, Cosaka ML, Romero EL, Morilla MJ. Uptake and intracellular traffic of siRNA dendriplexes in glioblastoma cells and macrophages. Int J Nanomedicine. 2011;6:2715-2728.

41. Nabi IR, Le PU. Caveolae/raft-dependent endocytosis. J Cell Biol. 2003;161(4):673-677.

42. Le Roy C, Wrana JL. Clathrin- and non-clathrin-mediated endocytic regulation of cell signalling. Nat Rev Mol Cell Biol. 2005;6(2): $112-126$.
43. Rejman J, Oberle V, Zuhorn IS, Hoekstra D. Size-dependent internalization of particles via the pathways of clathrin- and caveolae-mediated endocytosis. Biochem J. 2004;377(Pt 1):159-169.

44. Thomsen P, Roepstorff K, Stahlhut M, van Deurs B. Caveolae are highly immobile plasma membrane microdomains, which are not involved in constitutive endocytic trafficking. Mol Biol Cell. 2002;13(1): 238-250.

45. Anderson RG. The caveolae membrane system. Annu Rev Biochem. 1998;67:199-225.

46. Carver LA, Schnitzer JE. Caveolae: mining little caves for new cancer targets. Nat Rev Cancer. 2003;3(8):571-581.

47. Kobayashi H, Watanabe R, Choyke PL. Improving conventional enhanced permeability and retention (EPR) effects; what is the appropriate target? Theranostics. 2013;4(1):81-89. 


\section{Supplementary materials}

Table SI Zeta potentials and hydrodynamic diameters of LNPs formed at varying charge ratios

\begin{tabular}{lllll}
\hline $\begin{array}{l}\text { Charge } \\
\text { ratio }\end{array}$ & $\begin{array}{l}\text { Zeta potential } \\
\text { in OptiMEM } \\
(\mathrm{mV})\end{array}$ & $\begin{array}{l}\text { Hydrodynamic } \\
\text { diameter }(\mathrm{nm})\end{array}$ & $\begin{array}{l}\text { SD } \\
(\mathrm{nm})\end{array}$ & $\begin{array}{l}\text { Polydispersity } \\
\text { index }\end{array}$ \\
\hline $0.1 / 1$ & -11.5 & 262.1 & 124.5 & 0.198 \\
$0.5 / 1$ & -9.9 & 360.2 & 209.1 & 0.220 \\
$1 / 1$ & -9.6 & 385.7 & 158.8 & 0.124 \\
$3 / 1$ & -11.1 & 584.1 & 180.7 & 0.105 \\
$5 / 1$ & -6.19 & $1,126.0$ & 410.8 & 0.177 \\
\hline
\end{tabular}

Notes: While sizes of the complexes increase with increasing charge ratio, zeta potentials of complexes formed at different charge ratios do not vary significantly. Zeta potential, also known as the electrokinetic potential, is a measure of the charge of the electrical potential of a colloidal system with respect to the solution. Zeta potentials of all complexes were observed to be negative in OptiMEM (Life Technologies, Carlsbad, CA, USA) at $25^{\circ} \mathrm{C}$, in the -6 to $-12 \mathrm{mV}$ range, and there is no significant change in zeta potentials depending on the temperature of the environment (data not shown). In water, the complexes exhibit a slightly positive zeta potential of approximately $+18.5 \mathrm{mV}$; OptiMEM by itself has a zeta potential of $-30 \mathrm{mV}$; LNPs were formed with $50 \mathrm{nM}$ siRNA.

Abbreviations: LNPs, lipitoid-based nanoparticles; SD, standard deviation; siRNA, short interfering RNA.

A
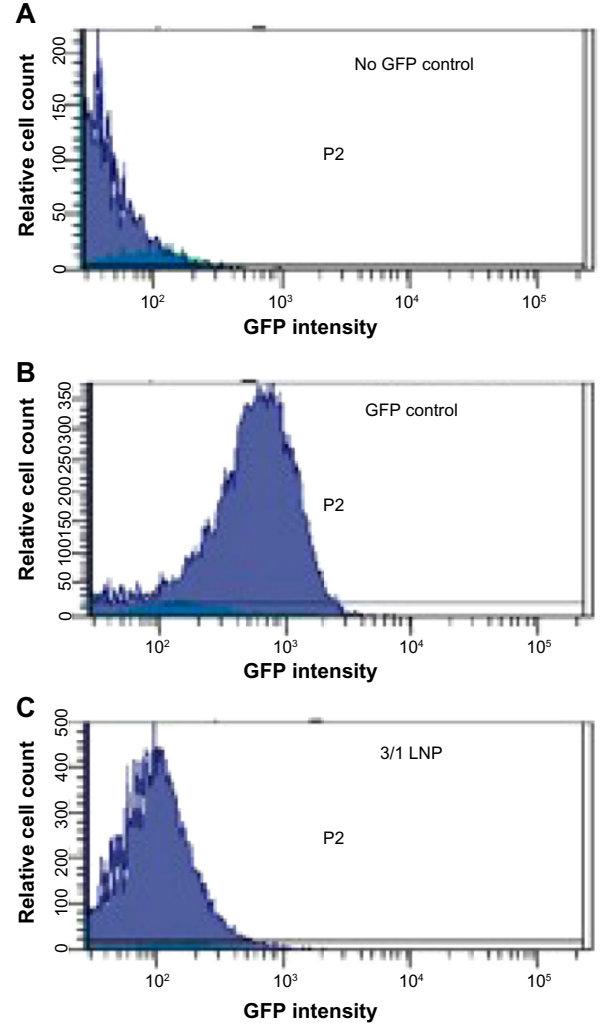

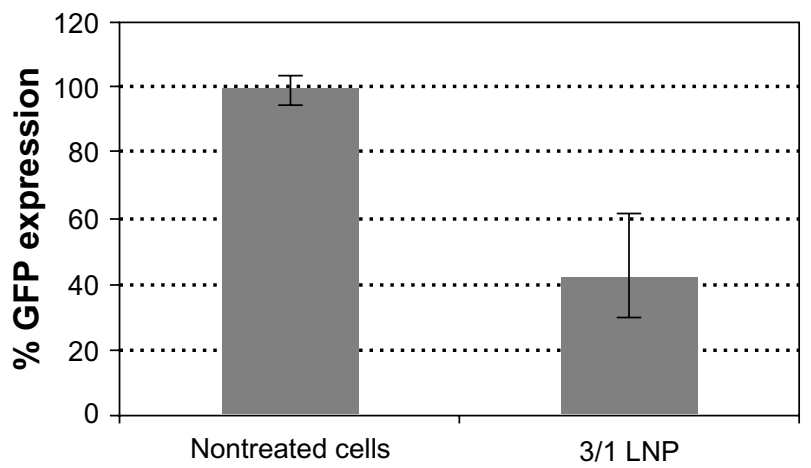

Figure SI Transfection of NM2C5 cells with siRNA-lipitoid LNPs.

Notes: Silencing of the GFP signal through the use of GFP siRNA and lipitoid based LNPs in GFP-expressing NM2C5 cells. The LNPs were formed at 3/I charge ratio and $50 \mathrm{nM}$ GFP siRNA was used. NM2C5 cells were viable throughout the experiment, and the LNPs were virtually non-toxic to NM2C5 cells unless the LNPs were formed above a charge ratio of $3 / 1$ (data not shown).

Abbreviations: GFP, green fluorescent protein; LNPs, lipitoid-based nanoparticles; siRNA, short interfering RNA.
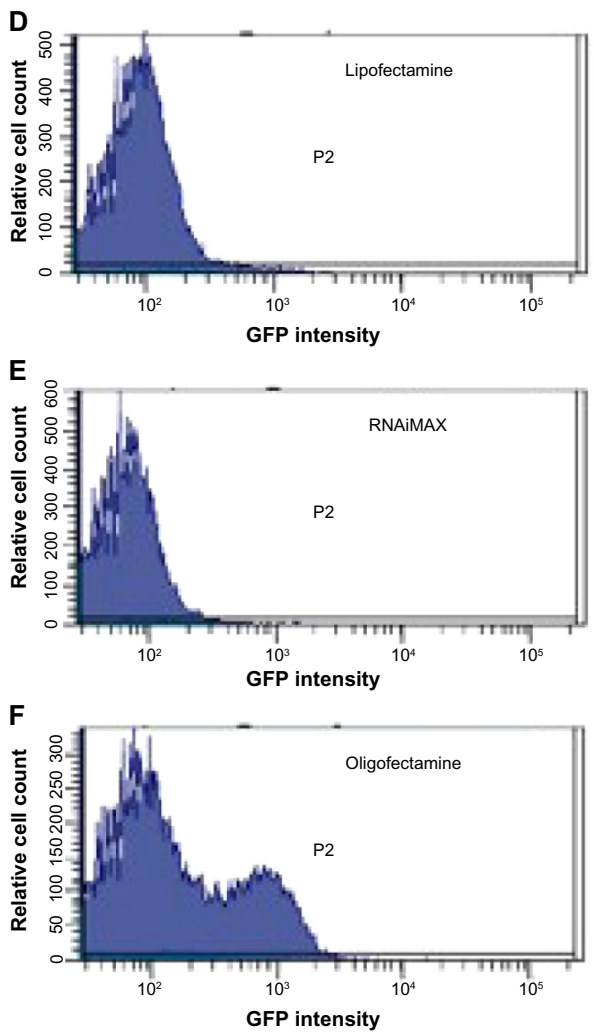

Figure S2 Silencing activity of LNPs is comparable to commercial transfection reagents.

Notes: FACS results showing the GFP silencing activity of the LNPs $(\mathbf{C})$ and the commercial reagents Lipofectamine ${ }^{\circledast}(\mathbf{D})$, RNAiMAX ${ }^{\mathrm{TM}}(\mathbf{E})$, and Oligofectamine $^{\mathrm{TM}}(\mathbf{F})$; Cells not expressing GFP (A) and GFP-expressing, nontreated cells (B) are shown as negative controls; LNPs were prepared with 50 nM siRNA, and all commercial reagents were used as instructed in the manufacturers' protocols (ranging from 20-200 nM siRNA), with transfection times ranging from 20 minutes to 4 hours; LNP-induced GFP silencing is comparable to Lipofectamine and RNAiMAX, and better than Oligofectamine.

Abbreviations: FACS, fluorescence assisted cell sorting; GFP, green fluorescent protein; LNPs, lipitoid-based nanoparticles; siRNA, short interfering RNA. 

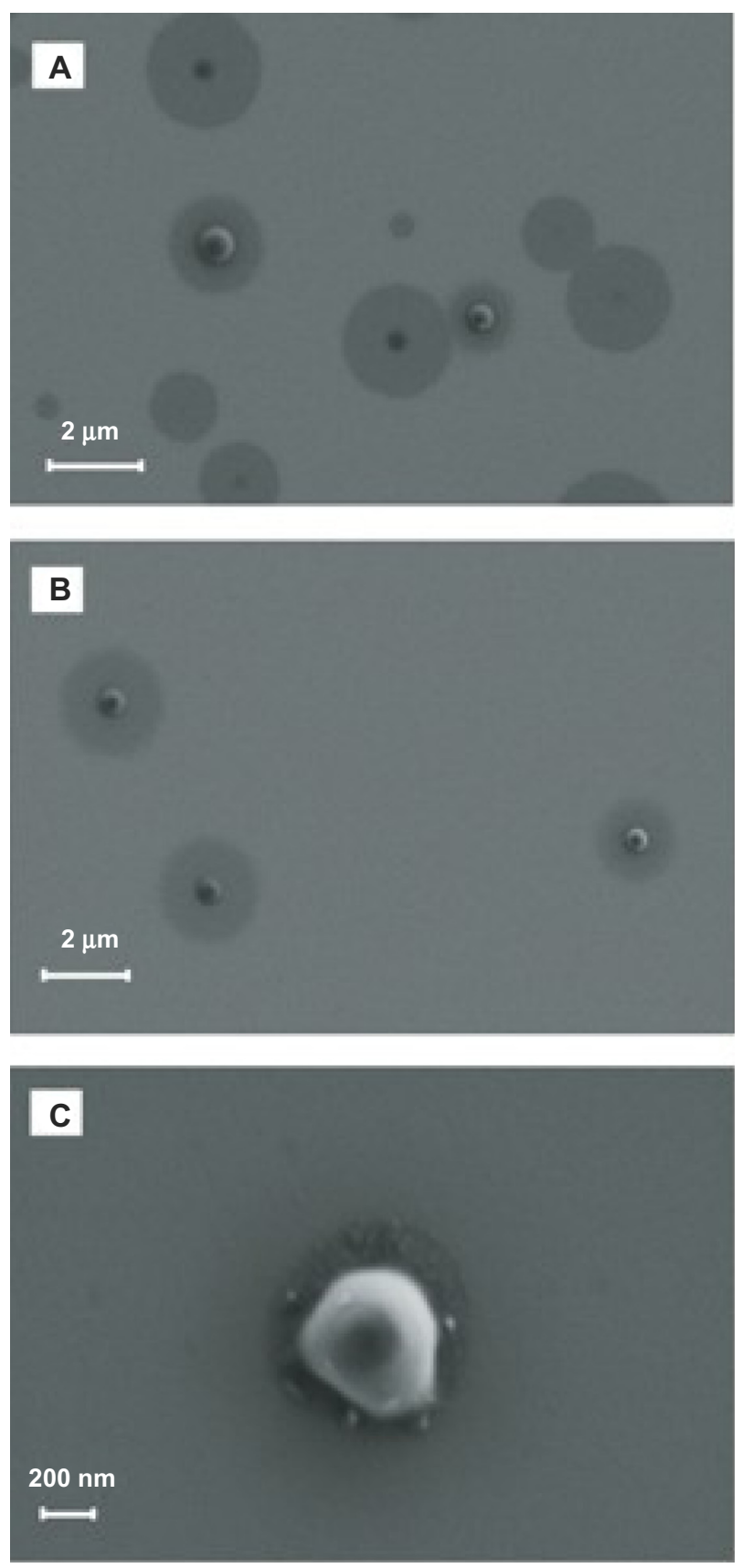

Figure S3 The effects on siRNA-lipitoid complexes 5 minutes after transferred into OptiMEM (A), 10 minutes after transferred into OptiMEM (B), and 60 minutes after transferred into OptiMEM (C).

Notes: To test whether complex morphology is affected not only by the environment of complex formation but also on the environment into which they are introduced, complexes of siRNA and lipitoid were formed at 3/I charge ratio in water, incubated for 10 minutes, then diluted in OptiMEM (Life Technologies, Carlsbad, CA, USA) $\mathrm{I} / \mathrm{I}$, and finally diluted $\mathrm{I} / \mathrm{IO}$ in OptiMEM before SEM imaging. Small complexes of around $200 \mathrm{~nm}$ in diameter were formed when complexes were formed in water. Upon dilution with OptiMEM, the complexes started to enlarge, forming a wide range of morphologies and started to resemble the complexes formed in OptiMEM (Figure S3.A). When these complexes were incubated in OptiMEM for 10 minutes, all the complexes became individual spheres of about $600 \mathrm{~nm}$ in diameter, with a more uniform size distribution (Figure S3.B). However, the overall number of observable complexes significantly decreased. In 60 minutes, fewer particles were observed, and the complexes start to deteriorate, losing their perfect spherical shape (Figure S3.C). In 90 minutes hardly any observable complexes were left (data not shown).

Abbreviations: SEM, scanning electron microscopy; siRNA, short interfering RNA.

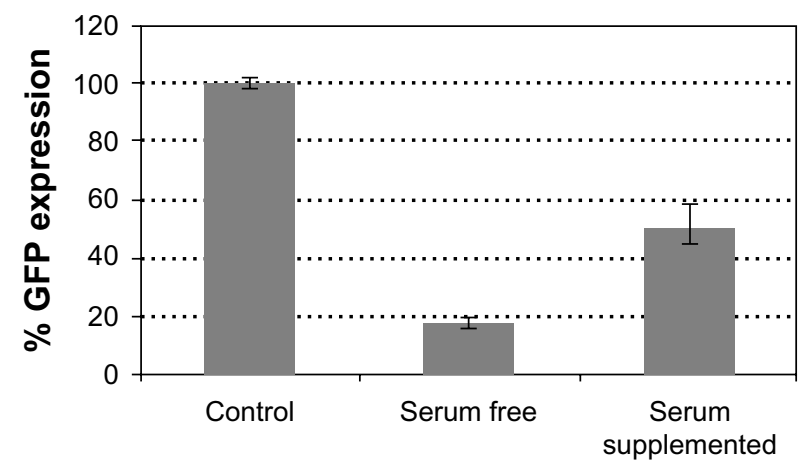

Figure S4 The effect of the presence of serum at the time of transfection on the silencing efficiency of LNPs.

Notes: LNPs formed at $3 / 1$ charge ratio were used in siRNA transfection of $50 \mathrm{nM}$ GFP siRNA onto GFP expressing U2OS cells incubated in either serum-free medium (only OptiMEM [Life Technologies, Carlsbad, CA, USA]) or serum-supplemented medium (OptiMEM supplemented with FBS to a final concentration of $10 \%$ serum). GFP levels decrease to $17.5 \% \pm 1.8 \%$ with serum-free medium as opposed to only $50.8 \% \pm 6.7 \%$ with serum-supplemented medium, revealing that the presence of serum at the time of transfection decreases the silencing efficiency of the LNPs, but still permits a significant silencing effect.

Abbreviations: FBS, fetal bovine serum; GFP, green fluorescent protein; LNPs, lipitoid-based nanoparticles; siRNA, short interfering RNA. 
International Journal of Nanomedicine

Dovepress

\section{Publish your work in this journal}

The International Journal of Nanomedicine is an international, peerreviewed journal focusing on the application of nanotechnology in diagnostics, therapeutics, and drug delivery systems throughou the biomedical field. This journal is indexed on PubMed Central, MedLine, CAS, SciSearch ${ }^{\circledR}$, Current Contents ${ }^{\circledR} /$ Clinical Medicine,
Journal Citation Reports/Science Edition, EMBase, Scopus and the Elsevier Bibliographic databases. The manuscript management system is completely online and includes a very quick and fair peer-review system, which is all easy to use. Visit http://www.dovepress.com/ testimonials.php to read real quotes from published authors.

Submit your manuscript here: http://www.dovepress.com/international-journal-of-nanomedicine-journal 